\title{
20 Apps for secondary school student mental health and wellbeing
}

\author{
Simone Gindidis and Mark Larsen
}

\section{How to use this policy}

Schools play an important role in supporting student mental health and wellbeing. Apps have fast become a useful tool to support young people's wellbeing due to the ubiquitous ownership and use of smartphone and tablet technology. Utilising apps to promote positive health and development enables schools to connect with students' increasingly digital needs. The development of policy to support the appropriate use of apps in this way is, therefore, necessary to encourage positive health outcomes and digital competence amongst students. This is only possible, however, if key stakeholders such as Allied Health and teaching staff are aware of the complexities associated with promoting responsible engagement with quality apps and avoid recommending poor quality or potentially harmful apps. School policy creators are encouraged to involve key stakeholders - including students - in this process. By adopting a school policy on apps for mental health and wellbeing, high-quality apps can be sourced for students who are most vulnerable to the potential risks of unchecked app use, such as accessing incorrect health information or unknowingly sharing personally identifiable information.

Updating and communicating effective policy on information and communication technologies is challenging due to the speed with which technology evolves. This policy example is predicated on the best available evidence at the time of writing and should be regularly reviewed to ensure technological, legal and ethical relevance. A policy on app use could be incorporated into a school-wide approach to personalised wellbeing practices and digital literacy curricula. This could be communicated on posters and infographics (Murray et al., 2017) made available to students in hardcopy format around campus wellbeing areas and through online mediums such as school social media or learning management systems. This policy should be considered alongside existing school mobile phone policy, particularly where exemptions can be granted for students using apps to manage symptoms associated with a mental health condition. This policy example is intentionally broad and school policy creators are encouraged to delete and build upon suggestions below to create a policy that best represents their school.

[To adapt and use this policy, delete or modify the text as indicated] 


\section{[INSERT name of school]'s use of apps for student mental health and wellbeing}

\section{Rationale}

Wellbeing teams in secondary schools play a pivotal role in supporting students to navigate a critical stage of life. Young people today appear to be navigating the same developmental challenges as those before them; however, they are now doing so within an increasingly technologically integrated world. The advent of the internet, smartphones and other forms of technology has fundamentally changed the way in which people are educated, communicate and work. Adolescents appear to favour the use of smartphones in particular: in 2015 it was estimated $80 \%$ of all Australian teenagers possess and use a smartphone (The Australian Communications and Media Authority, 2016). Smartphone apps, therefore, provide an avenue for wellbeing teams to facilitate unprecedented access to mental health information, assessment and interventions.

Young people are at risk of independently searching for and using poor quality apps containing inaccurate counselling guidance and advice (Furlonger \& Budisa, 2016). It is incumbent upon schools to ensure high quality, digitally relevant support tools are recommended to students that cater to the increasingly technological needs of young people.

\section{Purpose}

Young people often experience a number of barriers to accessing professional help for mental health issues. The purpose of this policy is to provide wellbeing and school leadership teams with research-informed guidance on the promotion of apps to support student mental health and wellbeing; specifically:

- Locating mental health and wellbeing apps

- Identifying high-quality apps

- Assessing app security and data collection practices

\section{Scope}

This policy applies to students, teachers, school leaders and Allied Health staff of [INSERT name of school]. This policy outlines important considerations for the recommendation and responsible use of apps for student wellbeing. By supporting the use of apps in this way, [INSERT name of school] is in no way advocating for student use of apps in lieu of accessing a mental health professional. Instead, [INSERT name of school] recognises the importance of equipping students and staff with evidence-informed guidance for how to identify apps that may assist students experiencing difficulties whilst they are seeking support from an appropriately trained professional.

[INSERT specific roles and responsibilities of individuals or teams] 


\section{Policy statement}

[INSERT name of school] acknowledges the crucial role played by schools to support the emotional and behavioural wellbeing of young people (Lawrence et al., 2015).

Apps are a form of mobile technology readily used by secondary schoolaged young people to communicate with one another and access information (Farmer, 2014; Madden et al., 2013; Roy Morgan Research, 2016; Taylor \& Silver, 2019). In the same way that educational apps and computing programs used at [INSERT name of school] provide students with the opportunity to rehearse and extend on classroom learning, apps can offer information and strategies to support students who may be experiencing symptoms associated with poor mental health such as stress, worry and sadness (Gibson et al., 2016; Gindidis et al., 2019b; 2019a; Wilansky et al., 2016).

There are a number of inherent challenges to using apps for mental health and wellbeing. These challenges primarily relate to data security, privacy and poor app design (Das et al., 2018; Gindidis et al., 2018; Grist et al., 2017; Kyrios et al., 2015; Larsen et al., 2019; Nicholas et al., 2015). Educators are encouraged to consider these limitations to ensure [INSERT name of school] promotes responsible use of low-risk apps for student wellbeing.

Studies suggest apps used in isolation are not as clinically effective as faceto-face interventions delivered by mental health practitioners. When required, staff will ensure students recommended apps for their mental health are also referred for internal, school-based support. [INSERT name of school] strongly recommends students engaging with apps for mental health and wellbeing consult a mental health professional such as a psychologist.

Privacy legislation relevant to [INSERT country where school is located] should also be reviewed to establish compliance when considering apps, for example, the 13 Australian Privacy Principles (APP) of the Privacy Act (1988) (Office of the Australian Information Commissioner, 2020).

\section{Reviewing apps}

[INSERT name of school] will ensure a member of staff reviews apps due to inherent difficulties with comprehension of privacy policies (Das et al., 2018). Unresolved privacy and security questions will be escalated to the App Developer and where necessary, [INSERT name of school]'s legal team or council.

[INSERT name of school] will consider reviewing apps not categorised as "mental health apps" such as music or exercise apps that have functions shown to promote positive health outcomes (Gindidis et al., 2018).

[INSERT name of school] will ensure the following key information is reviewed for each app to be recommended for student use:

- Intended audience (e.g., Was it designed for this age group?) 
- App developer (e.g., Was it developed by an academic or healthcare organisation, a commercial group, or a private individual?)

- Frequency of app updates (e.g., Apps last updated over six months ago may be associated with more quality concerns [Mercurio et al., 2020].)

- Privacy policy (e.g., does a policy actually exist? Are there assurances that data will not be shared with, or sold to, third parties? [Wu et al., 2017])

- Data collection practices (e.g., Does the app ask for personal identifiers such as name or date of birth, or integrate with Facebook? [Huckvale et al., 2019])

- Evidence to support app benefits (e.g., Apps may describe unreliable therapeutic approaches [Larsen et al., 2019] or may fail to faithfully implement evidence-based strategies [Huguet et al., 2016].)

- $\quad$ Ease of use (e.g., Poor usability is likely to lead to poor engagement with the app, and bugs and crashes can reduce confidence in the effectiveness of the app [Torous et al., 2018].)

[INSERT name of school] will refer to available app quality and security review frameworks to assess app quality, for example: the Mobile Application Rating Scale (Stoyanov et al., 2015) and the App Behaviour Change Scale (McKay et al., 2019).

\section{Recommending apps to students}

[INSERT name of school] will only recommend apps which are downloaded through official app stores (e.g., Apple App Store, Google Play Store) due to risks associated with apps from other unofficial sources (The OWASP Foundation, 2020).

Wellbeing staff at [INSERT name of school] will ensure apps recommended for student wellbeing and mental health include evidence-based behaviour change strategies, such as: psychoeducation, goal setting, self-monitoring, coping planning, relaxation and problem solving (Australian Psychological Society, 2018; Gindidis et al., 2018; Kallapiran et al., 2015; Lukens \& McFarlane, 2004; Michie et al., 2013; Rickwood et al., 2005).

[INSERT name of school] will not recommend apps based on app store ratings (Nicholas et al., 2015) or testimonials (Larsen et al., 2019) as these are not good indicators of app quality.

[INSERT name of school] will only recommend to students high-quality apps that have been deemed safe. Determination of app quality and safety will be based on the key information criteria outlined in the "Reviewing apps" section of this policy.

\section{Staff and students}

Student digital literacy is a core skill in today's world and at [INSERT name of school] every effort is made to embed technology across the school's curriculum - including student welfare. 
Students of [INSERT name of school] will be encouraged to develop the skills necessary to undertake app quality and security reviews in an effort to limit the gap between policy and competency (Jacobson et al., 2010; Porat et al., 2018). Student-led activities such as group projects will involve exploration of the key quality indicators previously outlined relating to app security, user experience, privacy and evidence. Activities will be embedded within relevant learning areas such as legal studies, civics and citizenship, and computer studies.

\section{Rating of evidence base}

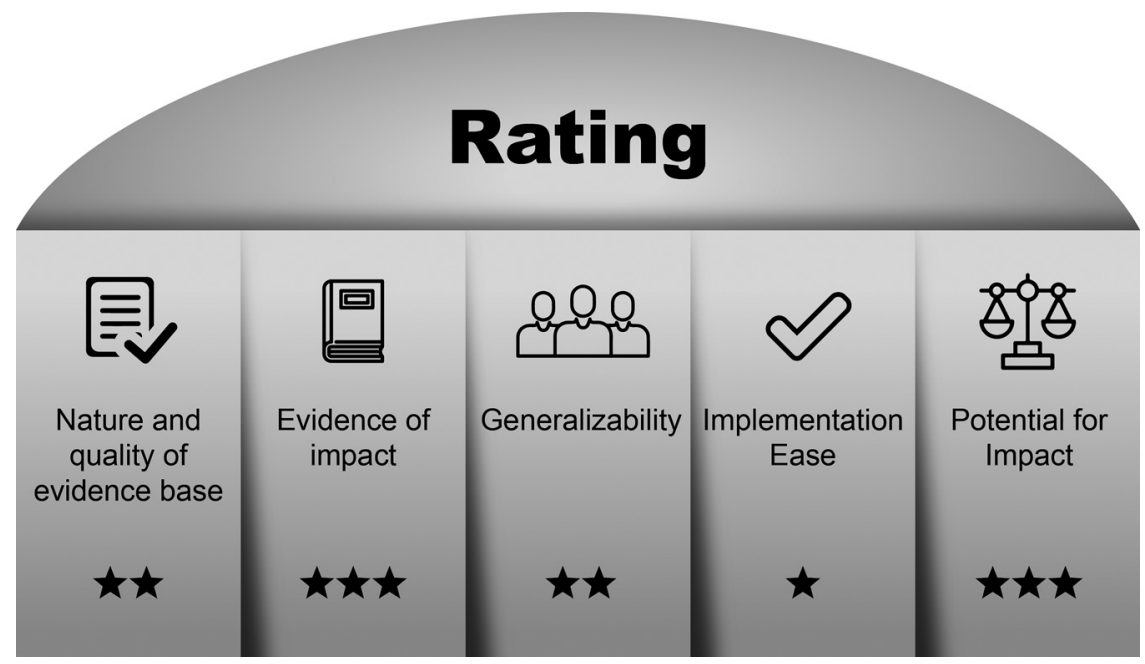

Figure 20.1. Apps for Secondary School Student Mental Health and Wellbeing Rating of Evidence.

Author Note. Research indicates that young people are increasingly expressing a preference for engaging with mobile wellbeing and mental health supports. Evidence of app efficacy is mixed and possibly biased, with app developers and researchers often one and the same. Although quality apps are in the minority, studies of high-quality, evidence-informed and co-designed apps are suggestive of positive engagement and outcomes. Smartphone ownership and app download metrics indicate that apps offer young people on-demand access to information and adjunctive wellbeing support. Young people's ability to discern high-quality apps is an important skill currently lacking explicit integration into school curriculums. Evidence used to inform this policy is not informed by studies that consider school contexts. Implementation of this policy is predicated on the digital competency of staff. Unlike many other school policies, this policy will need to be regularly reviewed to ensure compliance with evolving legislation and technology. App recommendations should be updated periodically to ensure continuing relevance and appropriateness with app updates. Policy enactment will not involve significant funds. In addition to being used by school wellbeing staff, appraisal of mental health and wellbeing apps can also potentially be embedded across digital citizenship and health curricula. 


\section{Authorship}

Dr Simone Gindidis, Monash University

Dr Mark Larsen, Black Dog Institute, University of New South Wales

[INSERT RELEVANT STAFF MEMBERS]

\section{Related policy and documents}

Mobile phone policy

Student wellbeing policy

Health and wellbeing services

Electronic devices policy

Safety policy

Privacy statement

Counselling policy

[INSERT RELEVANT POLICY AND DOCUMENTS]

\section{Date of ratification}

This policy was ratified on the [INSERT DATE].

\section{Date of review}

This policy will be reviewed by [INSERT DATE].

\section{Further reading}

American Psychiatric Association. (2018). App evaluation model. https://www. psychiatry.org/psychiatrists/practice/mental-health-apps/app-evaluation-model

Department of Health. (2020). Head to health. https://headtohealth.gov.au/

Department of Health and Social Care. (2020) NHS Apps Library.https:// www.nhs.uk/apps-library/

One Mind. (2020). PsyberGuide. www.psyberguide.org

Orcha. (2020). COVID-19 Health Apps. https://covid19.orcha.co.uk/

Reach Out. (2020). Tools and Apps.https://au.reachout.com/tools-and-apps

\section{Expert consultation}

School information technology, computing and/or technical teams

Department of Education (or equivalent) legal team

School allied health and wellbeing professionals

\section{References}

Australian Psychological Society. (2018). Evidence-based psychological interventions in the treatment of mental disorders: A literature review. Australian Psychological Society Ltd. 
Das, G., Cheung, C., Nebeker, C., Bietz, M., \& Bloss, C. (2018). Privacy policies for apps targeted toward youth: Descriptive analysis of readability. JMIR MHealth and UHealth, 6(1), e3.

Farmer, A. (2014). A third of mobile-owning teens also "own" a tablet. YouGov UK. https://yougov.co.uk/news/2014/02/04/third-mobile-owning-teens-also-own-tablet/

Furlonger, B., \& Budisa, S. (2016). Internet sites and apps available to students seeking counselling, and what school counsellors should know about them. Journal of Psychologists and Counsellors in Schools, 26(1), 68-83.

Gibson, K., Cartwright, C., Kerrisk, K., Campbell, J., \& Seymour, F. (2016). What young people want: A qualitative study of adolescents' priorities for engagement across psychological services. Journal of Child and Family Studies, 25, 1057-1065.

Gindidis, S., Stewart, S. E., \& Roodenburg, J. (2019a). Adolescent experiences of appintegrated therapy. Educational and Developmental Psychologist, 37(1), 20-29.

Gindidis, S., Stewart, S. E., \& Roodenburg, J. (2019b). Psychologists' motivations for integrating apps into therapy with secondary school-aged young people. Journal of Psychologists and Counsellors in Schools, 30(1), 2-12.

Gindidis, S., Stewart, S. E., \& Roodenburg, J. (2018). A systematic scoping review of adolescent mental health treatment using mobile apps. Advances in Mental Health, 17(2), 161-171.

Grist, R., Porter, J., \& Stallard, P. (2017). Mental health mobile apps for preadolescents and adolescents: A systematic review. Journal of Medical Internet Research, 19(5), e176.

Huckvale, K., Torous, J., \& Larsen, M. E. (2019). Assessment of the Data Sharing and Privacy Practices of Smartphone Apps for Depression and Smoking Cessation. JAMA Network Open, 2(4), e192542-e192542.

Huguet, A., Rao, S., McGrath, P. J., Wozney, L., Wheaton, M., Conrod, J., \& Rozario, S. (2016). A Systematic Review of Cognitive Behavioral Therapy and Behavioral Activation Apps for Depression. PLOS ONE, 11(5), e0154248.

Jacobson, M. J., So, H.-J., Teo, T., Lee, J., Pathak, S., \& Lossman, H. (2010). Epistemology and learning: Impact on pedagogical practices and technology use in Singapore schools. Computers \& Education, 55, 1694-1706.

Kallapiran, K., Koo, S., Kirubakaran, R., \& Hancock, K. (2015). Review: Effectiveness of mindfulness in improving mental health symptoms of children and adolescents: A meta-analysis. Child and Adolescent Mental Health, 20(4), 182-194.

Kyrios, M., Abbott, J., Reynolds, J., \& Thomas, N. (2015). Ethical aspects of psychological assessment, treatment and research over the internet. In S. Morrissey, P. Reddy, \& G. Davidson (Eds.), Ethics and professional practice for psychologists (2nd ed.)(pp. 122-133). Cengage Learning Australia.

Larsen, M. E., Huckvale, K., Nicholas, J., Torous, J., Birrell, L., Li, E., \& Reda, B. (2019). Using science to sell apps: Evaluation of mental health app store quality claims. NPJ Digital Medicine, 2(18).

Lawrence, D., Johnson, S., Hafekost, J., de Haan, K. B., Sawyer, M., Ainley, J., \& Zubrick, S. R. (2015). The mental health of children and adolescents: Report on the second Australian child and adolescent survey of mental health and wellbeing. http:// www.health.gov.au/internet/main/publishing.nsf/Content/9DA8CA21306 FE6EDCA257E2700016945/\$File/child2.pdf

Lukens, E. P., \& McFarlane, W. R. (2004). Psychoeducation as evidence-based practice: Considerations for practice, research, and policy. Brief Treatment \& Crisis Intervention, 4(3), 205-225. 
Madden, M., Lenhart, A., Cortesi, S., \& Gasser, U. (2013). Teens and mobile apps. Pew Research Center.

McKay, F. H., Slykerman, S., \& Dunn, M. (2019). The App Behavior Change Scale: Creation of a scale to assess the potential of apps to promote behavior change. JMIR Mhealth Uhealth, 7(1), e11130.

Mercurio, M., Larsen, M., Wisniewski, H., Henson, P., Lagan, S., \& Torous, J. (2020). Longitudinal trends in the quality, effectiveness and attributes of highly rated smartphone health apps. Evidence Based Mental Health, 23(2), 107-111.

Michie, S., Richardson, M., Johnston, M., Abraham, C., Francis, J., Hardeman, W., Eccles, M., Cane, J., \& Wood, C. (2013). The Behavior Change Technique Taxonomy (v1) of 93 hierarchically clustered techniques: Building an international consensus for the reporting of behavior change interventions. Annals of Behavioral Medicine: A Publication of the Society of Behavioral Medicine, 46(1), 81-95.

Murray, I. R., Murray, A. D., Wordie, S. J., Oliver, C. W., Murray, A. W., \& Simpson, A. H. R. W. (2017). Maximising the impact of your work using infographics. Bone \& Joint Research, 6(11), 619-620.

Nicholas, J., Larsen, M. E., Proudfoot, J. G., \& Christensen, H. (2015). Mobile apps for bipolar disorder: A systematic review of features and content quality. Journal of Medical Internet Research, 17(8), e198.

Office of the Australian Information Commissioner. (2020). Australian Privacy Principles quick reference. https://www.oaic.gov.au/privacy/australian-privacy-principles/ australian-privacy-principles-quick-reference/

Porat, E., Blau, I., \& Barak, A. (2018). Measuring digital literacies: Junior high-school students' perceived competencies versus actual performance. Computers \& Education, 126, 23-36.

Rickwood, D., Deane, F. P., Wilson, C. J., \& Ciarrochi, J. (2005). Young people's helpseeking for mental health problems. Australian E-Journal for the Advancement of Mental Health, 4(3), 218-251.

Roy Morgan Research. (2016, August 22). Article No. 6929: 9 in 10 Aussie teens now have a mobile (and most are already on to their second or subsequent handset). Roy Morgan Research. http://www.roymorgan.com/findings/6929-australian-teenagersand-their-mobile-phones-june-2016-201608220922

Stoyanov, S., Hides, L., Kavanagh, D. J., Zelenko, O., Tjondronegoro, D., \& Mani, M. (2015). Mobile app rating scale: A new tool for assessing the quality of health mobile apps. JMIR MHealth UHealth, 3(1), e27

Taylor, K., \& Silver, L. (2019). Smartphone ownership is growing rapidly around the world, but not always equally. PEW Research Center. https://www.pewresearch.org/ global/wp-content/uploads/sites/2/2019/02/Pew-Research-Center_Global-TechnologyUse-2018_2019-02-05.pdf

The Australian Communications and Media Authority. (2016). Aussie teens and kids online. http://www.acma.gov.au/theACMA/engage-blogs/engage-blogs/Researchsnapshots/Aussie-teens-and-kids-online

The OWASP Foundation. (2020). OWASP mobile top ten. https://owasp.org/wwwproject-mobile-top-10/

Torous, J., Nicholas, J., Larsen, M. E., Firth, J., \& Christensen, H. (2018). Clinical review of user engagement with mental health smartphone apps: Evidence, theory and improvements. Evidence Based Mental Health, 21(3), 116.

Wilansky, P., Eklund, J. M., Milner, T., Kreindler, D., Cheung, A., Kovacs, T., Shahin, S., Astell, A., Ohinmaa, A., Henderson, J., Strauss, J., \& Mills, R. S. (2016). 
Cognitive behavior therapy for anxious and depressed youth: Improving homework adherence through mobile technology. JMIR Research Protocols, 5(4), e209.

Wu, E., Torous, J., Hardaway, R., \& Gutheil, T. (2017). Confidentiality and Privacy for Smartphone Applications in Child and Adolescent Psychiatry: Unmet Needs and Practical Solutions. Health Information Technology for Child and Adolescent Psychiatry, 26(1), 117-124. 